\title{
Análisis de dos modelos de Terapia Ocupacional a partir de la noción de modelo en la Filosofía de la Ciencia ${ }^{1}$
}

\author{
Analysis of two Occupational Therapy models from the \\ Philosophy of Science's notion of model
}

\author{
Jaqueline Cruz Perdomo ${ }^{2}$ \\ Recibido: 26 junio 2015 • Enviado para modificación: 9 julio 2015 • Aceptado: 16 julio 2015. \\ Cruz, J. (2015). Análisis de dos modelos de Terapia Ocupacional a partir de la noción de modelo en la Filosofía de la Ciencia. \\ Revista Ocupación Humana, 15 (1), pp. 35 - 47.
}

\begin{abstract}
Resumen: Se busca comprender las ideas de modelo y de teoría en las cuales se enmarcan los conocimientos de Terapia Ocupacional, proponiendo un diálogo con la Filosofía de la Ciencia, en relación específica con su noción de modelo y teoría en el enfoque semántico - modelista. El análisis responde a la pregunta ¿Qué tipo de modelos son el Modelo Desempeño Ocupacional Realizante y el Modelo de Ocupación Humana, a la luz de una tipología de la noción de modelo de la Filosofía de la Ciencia?. Del análisis se desprende que los dos modelos se comportan como tales, según el enfoque modelista de la Filosofía de la Ciencia, dado que poseen una estructura conceptual de tipo simbólica que idealiza la noción de ocupación humana o desempeño ocupacional; además, cumplen la función de representación simbólica. Por lo tanto, estos dos modelos en Terapia Ocupacional se comportan como modelos representacionales de tipo simbólico no matemático, asimilables a los modelos teóricos de la Filosofía de la Ciencia.
\end{abstract}

Palabras clave: Conocimiento, Terapia Ocupacional, Filosofía de la Ciencia.

Abstract: This article aims for an understanding of which ideas of the notions of model and theory frame knowledge in Occupational Therapy. Thus, the author proposes a dialogue with the philosophy of science in specific relation with its notion of model within the semantic approach of theory. The analysis answers the question: what type of model are the 'model of fulfilling occupational performance' and 'the model of human occupation', in the light of a typology of the notion of model of the philosophy of science? The author concludes that both models behave like such. According to the modeling approach of the philosophy of science, they both have a type of symbolic structure that idealizes the notion of human occupation or occupational performance. Besides, these models accomplish the function

\footnotetext{
${ }^{1}$ Este artículo se basa en el trabajo de grado "Tipología de la noción de modelo y su aplicación a dos modelos en Terapia Ocupacional", realizado por la autora para optar el título de Magíster en Filosofía, en el año 2012.

${ }^{2}$ Terapeuta Ocupacional. Magíster en Filosofía. Estudiante Doctorado Interinstitucional en Educación. Profesora Terapia Ocupacional. Universidad del Valle. Cali, Colombia. jaqueline.cruz@correounivalle.edu.co
} 
of symbolic representation. In Occupational Therapy, they act as representational models of a symbolic, not mathematic nature, similar to the theoretical models of the philosophy of science.

Key words: Knowledge, Occupational Therapy, Philosophy of science.

\section{La apuesta por comprender la noción de modelo}

En Terapia Ocupacional se usan varias denominaciones para dar cuenta de un cuerpo teórico relacionado con una práctica; el modo como se presenta esta relación, desde diversas posiciones, es bajo la denominación de modelo. En este escrito se aborda el análisis de dos propuestas específicas respecto a esta noción en Filosofía de la Ciencia, a partir de los trabajos de Trujillo, desde 1992 a 1994, y de Kielhofner, en 2004 y 2006, y sus Modelo del Desempeño Ocupacional Realizante y Modelo de la Ocupación Humana, respectivamente.

Las relaciones que se puedan establecer, entre los conceptos que se usan en Terapia Ocupacional y los que se usan en Filosofía de la Ciencia, pueden contribuir a la problematización y enriquecimiento crítico de la identidad sobre los modos de construir y expresar el conocimiento dentro del ejercicio específico de disciplinas. La Terapia Ocupacional tiene cuerpos teóricos y metodológicos conjugados cuya comprensión es importante, no sólo para el trabajo profesional, sino para la cualificación de la construcción conceptual que los sustenta.

Tanto por su relevancia académica en Terapia Ocupacional, como por el lugar de la práctica personal de quien realiza este estudio, como formadora de recurso humano en esta profesión, el Modelo del Desempeño Ocupacional Realizante de Trujillo (1994, 1995) y el Modelo de Ocupación Humana de Kielhofner (2004) constituyen los referentes de análisis y reflexión conceptual de este documento.

El de Trujillo es el primer modelo para la comprensión de la Terapia Ocupacional construido en Colombia, a partir de una serie de estudios realizados en la década del 80 sobre los modelos usados por los terapeutas ocupacionales en el país. Presenta una idea de desempeño ocupacional como realización humana que se consigue cuando las personas logran un dominio competente sobre sus ocupaciones. Entre los propósitos de este modelo está el de ser un referente para la formación universitaria en el país (TrujiIlo, 1994). Un ejemplo de esto es el currículo de Terapia Ocupacional de la Universidad del Valle (Resolución del Consejo Académico 053 de febrero 26 de 2004), que contiene la noción de desempeño ocupacional a través de las líneas de formación: procesos ocupacionales, procesos humanos, interacción con el medio y conceptualización del desempeño ocupacional.

Por su parte, el Modelo de Ocupación Humana aparece reseñado en la mayoría de la literatura internacional y nacional de Terapia Ocupacional. 
Es un modelo de alto reconocimiento académico y es comúnmente utilizado en la práctica por los terapeutas. Centra su mirada en comprender a las personas como seres ocupacionales, siendo su tesis central que los seres humanos tienen una naturaleza innata de tipo ocupacional. Por su continua producción investigativa, este modelo se ha consolidado en los argumentos teóricos, así como en la tecnología para la intervención terapéutica. Se considera de obligatoria enseñanza en los currículos de las diferentes universidades del mundo que forman terapeutas ocupacionales.

Otra de las razones para estudiar este tema está relacionada con una inquietud personal desde mi práctica como profesora de Terapia Ocupacional, dado que esta construye, divulga y usa modelos en la intervención ocupacional.

Este trabajo pretende aportar a la rigurosidad en la construcción del conocimiento y a su uso en la Terapia Ocupacional, en la medida en que se pregunta si los modelos estudiados son entendidos como idealizaciones teóricas y qué tipo de modelo son a la luz de la tipología de noción de modelo en ciencia.

\section{Los modelos y el enfoque semántico-modelista de las teorías}

El uso y la construcción de modelos para conocer y usar el mundo son prácticas culturales y cognitivas de universal arraigo en el ejercicio humano de satisfacer la necesidad de comprender para hacer. Se modela la realidad de muchas y variadas maneras, con fines y usos igualmente diversos: desde ideas de modelo como ejemplo a seguir (por ejemplo, se infunde a los hijos seguir el buen ejemplo de sus padres), pasando por la instauración cultural de algo como modelo de algo (ejemplo, la muñeca Barbie como modelo de belleza en occidente), hasta modos específicos y más elaborados de representación, como el retrato de un artista, un diagrama del sistema solar, el plano de instalaciones hidráulicas de una casa, el mapa del zoológiCO, una gráfica estadística sobre la tendencia de homicidios en una ciudad, un enunciado de la lógica formal, una teoría, etc.

Este trabajo busca analizar dos modelos de Terapia Ocupacional, a partir de la noción de modelo que se trabaja en el enfoque semántico - modelista de la Filosofía de la Ciencia, que ha trabajado en la comprensión del conocimiento que crean los científicos y en las formas en las que lo construyen; entre los problemas que aborda están las nociones de teoría y de modelo presentes en la construcción de conocimiento. Algunos de los autores representantes de esta discusión filosófica son Max Black (1966), Ronald Giere (1999) y Germán Guerrero (2010).

Para Giere (1999), los modelos que construyen los científicos son representaciones que se hacen del mundo para comprenderlo. Entre el modelo o la familia de modelos y el sistema real se establece una relación de semejanza en ciertos aspectos y grados, por mediación de las hipótesis teóricas. A su vez, se da otra relación de verdad o falsedad entre el sistema real y las hipótesis teóricas. El autor aclara que los modelos usan palabras y diagramas 
para expresarse. Esto no implica que sean entidades lingüísticas, sino que se expresan a través del lenguaje. Por su parte, las hipótesis teóricas sí representan entidades lingüísticas, enunciados que afirman cierto tipo de relación entre el modelo y el sistema real.

De los autores mencionados se toma la noción de teoría del enfoque semántico-modelista, en el que se entiende que una teoría es una entidad abstracta, un conjunto de modelos, de estructuras que representan parcelas del mundo en ciertos aspectos y grados. Así pues, una teoría queda mejor entendida como un conjunto de modelos.

Las características del enfoque semántico - modelista, en relación con la noción de teoría, de acuedro a Guerrero (2010), se sintetizan de la siguiente manera:

- La teoría es un conjunto de modelos, estructuras que idealizan constructos acerca del mundo, es un objeto abstracto.

- Los principios representan un modelo y la relación de la teoría con el mundo es de aproximación.

- La relación con el mundo es de adecuación o aproximación, dado que es una representación en ciertos aspectos y grados.

- La teoría es una representación parcial y aproximada del mundo.

Ahora bien, los modelos se pueden clasificar en dos grandes categorías: Modelos representacionales y Modelos no representacionales (como se sintetizan en la Tabla 1).

\section{Modelos representacionales}

La concepción representacional de los modelos se ubica dentro del enfoque semántico-modelista para la comprensión de una teoría. Su principal característica es que la estructura o el modelo cumple la función de representar al mundo en ciertos aspectos y grados. Como describe Giere (1999), "Ilamo representacional a la forma como entiendo los modelos, porque no los considero como proporcionando un medio para interpretar sistemas formales, sino más bien como herramientas para representar el mundo" (p.3).

En cuanto a la característica de su función representacional, algunos modelos son de carácter pictórico, como los son: el modelo icónico, modelo a escala, modelo analógico, mapas, diagramas y arquetipos. Otros, en cambio, son de carácter simbólico, como el modelo matemático aplicado o el modelo teórico.

En los modelos pictóricos la relación de representación entre el modelo y el modelo del mundo es de isomorfismo en la estructura, ya que esta se conserva. En cuanto a los modelos de carácter simbólico, debe destacarse que la relación de representación entre el modelo y el modelo del mundo no es de identidad en la estructura, sino de relación entre un lenguaje que idealiza los objetos del mundo y estos propiamente, de tal manera que la representación simboliza pero no replica lo representado.

\section{Modelos no representacionales}

Los modelos no representacionales se ubican dentro del enfoque semántico 
Tabla 1. Clasificación de modelos representacionales y no representacionales

\begin{tabular}{|c|c|c|c|}
\hline \multicolumn{2}{|c|}{ Modelos representacionales } & \multicolumn{2}{|c|}{ Modelos no representacionales } \\
\hline Pictóricos & Simbólicos & Interpretativos & $\begin{array}{l}\text { Satisface una } \\
\text { descripción }\end{array}$ \\
\hline Modelo icónico & $\begin{array}{l}\text { Modelo matemático } \\
\text { aplicado }\end{array}$ & \multirow[t]{6}{*}{$\begin{array}{l}\text { Modelo semántico } \\
\text { o lógico }\end{array}$} & $\begin{array}{l}\text { Modelo matemático } \\
\text { puro }\end{array}$ \\
\hline Modelo a escala & \multirow[t]{5}{*}{ Modelo teórico } & & \multirow{5}{*}{$\begin{array}{l}\text { Modelo ajustado a } \\
\text { una descripción }\end{array}$} \\
\hline Modelo analógico & & & \\
\hline Mapas & & & \\
\hline Diagramas & & & \\
\hline Arquetipos & & & \\
\hline
\end{tabular}

Fuente. Elaboración propia a partir de las tipologías de la noción de modelo de los trabajos de Black (1966), Giere (1999) y Guerrero (2010).

en su versión estructural de las teorías. Su principal característica es que una determinada axiomatización conjuntista informal se satisface o es verdadera en el modelo. Los hay de tres tipos: modelos semánticos o interpretativos, modelos matemáticos puros y modelos que se ajustan a una descripción. Estos modelos son no representacionales porque el predicado conjuntista se interpreta en la estructura que describe el modelo teórico.

\section{Análisis de dos modelos en Terapia Ocupacional}

\section{El Modelo Desempeño Ocupacional Realizante}

En los años 80 tuvo origen el Modelo Desempeño Ocupacional Realizante, producto de la reflexión de profesoras de Terapia Ocupacional de la Universidad Nacional de Colombia (Trujillo, 1994). Surge en un momento histórico en el que confluyen, en el contexto académico colombiano, la necesidad de hacer una explicación que integre las dimensiones biológica y sociocultural del sujeto que desempeña una ocupación, y la de repensar el objeto de estudio como posibilidad de identidad y guía para la enseñanza universitaria y el ejercicio profesional. Todo esto se tradujo en una respuesta de teorización sobre el conocimiento en la terapia, materializada en este modelo, cuyo planteamiento central sustenta que el desempeño ocupacional se configura como producto de la interacción entre los procesos humanos y ocupacionales, influidos por el medio ambiente.

El desempeño ocupacional en sus categorías de autocuidado, juego y trabajo es una constante histórica en el ejercicio de la Terapia Ocupacional. Por lo tanto, es necesario concebir una estructura que represente ese concepto de manera dinámica... La estructura debe ser tal que permita: a) guiar el proceso educativo; b) 
orientar la identidad ocupacional de profesores, estudiantes y egresados; c) encauzar la investigación que requieren la profesión y la sociedad. (Trujillo, 1994, p. 101)

Desde una perspectiva humanistaexistencial, este modelo concibe el actuar ocupacional como dominio de estudio de la Terapia Ocupacional y conjunto de acciones a través de las cuales las personas tienden a su realización. Así pues, "El modelo desempeño ocupacional realizante simboliza el dominio de estudio de Terapia Ocupacional, con énfasis en sus propósitos. La estructura prioriza la direccionalidad de la Terapia Ocupacional e igualmente corresponde a los propósitos del actuar ocupacional del hombre" (Trujillo, 1995, p. 11).

El Desempeño Ocupacional Realizante representa el actuar del hombre motivado por sus propias necesidades. Estas pueden potencialmente ser satisfechas a través de la realización de actividades de autocuidado, juego y trabajo, de acuerdo con sus recursos personales y las características del contexto. Por lo tanto, posee un carácter ascendente al promover la organización e integración, productividad y realización de la persona en su vivencia ocupacional. De esta forma,

...el desempeño ocupacional realizante es un modelo conceptual que se refiere a la experiencia de avanzar en el proceso de realización personal. El modelo surge de la integración de tres dimensiones: los procesos humanos, los procesos ocupacionales y el contexto físico, temporal y sociocultural...El modelo postula que la interrelación de estas dimensiones se inicia cuando la persona, en cada contexto particular, guiada por sus necesidades de subsistencia, socialización, dominio y actualización, se compromete con las ocupaciones de autocuidado, juego y trabajo. A esta conjugación se le denomina desempeño ocupacional realizante, el cual involucra: a) las necesidades y capacidades individuales; b) los procesos ocupacionales seleccionados por las personas; y c) los estímulos del contexto físico, temporal y sociocultural en el cual la experiencia adquiere significado. El modelo postula que la persona, al actuar ocupacionalmente, tiende a su propia realización, integración y productividad, los cuales son ganancias esenciales para el ascenso del ser humano. (Trujillo, 1995, p. 13)

Sus autoras lo clasifican como un modelo conceptual porque, desde una posición teórica integral, pone en diálogo teorías biológicas, psicológicas, sociales, antropológicas y culturales para describir y comprender al sujeto, las ocupaciones que realiza y el contexto donde las desarrolla. En la Figura 1 se representan los elementos estructurales que lo caracterizan, en sus versiones de 1992 y 1994.

Como se detalla en la Figura 1, el modelo propone que el dominio conceptual que explica el quehacer del terapeuta ocupacional se encuentra en el espacio dinámico formado por la confluencia de dos dimensiones o coordenadas: procesos humanos y procesos ocupacionales. Estos surgen y se mueven en un ambiente espaciotemporal y social.

Los procesos humanos se entienden como niveles crecientes de satisfac- 
Figura 1. Representaciones del Modelo Desempeño Ocupacional Realizante, versiones 1992 y 1994
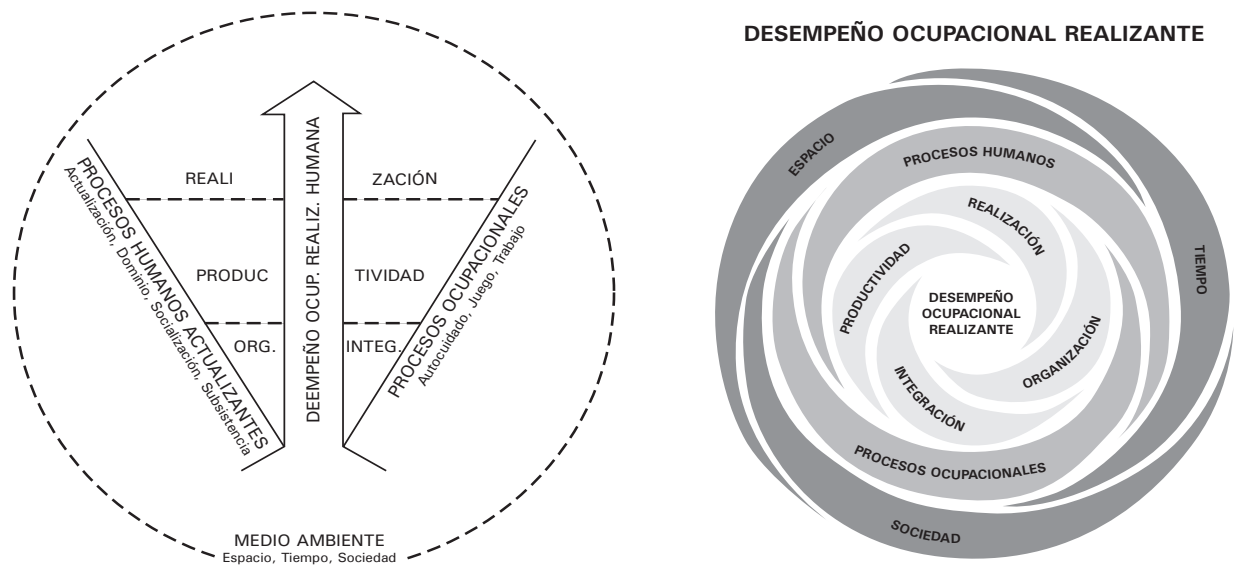

Fuente. Trujillo (1994, p. 103) y Trujillo (1995, p. 12).

ción de necesidades como subsistencia, socialización, dominio y actualización, sustentados en el potencial sensorial, motor, cognoscitivo, afectivo y social de la persona. Por su parte, los procesos ocupacionales están representados por el autocuidado, el juego y el trabajo, y sus componentes neuromotor, integrativo, sensorial, cognoscitivo, emocional y social.

El medio ambiente está constituido por "un espacio, una temporalidad y una estructura social, económica e ideológica que favorece o impide que el desempeño ocupacional tienda a la realización de la persona" (Trujillo, 1994, p. 102).

\section{El Modelo Ocupación Humana}

Alrededor de 1980, un grupo de terapeutas ocupacionales vuelve a pensar la identidad de la terapia y su objeto de conocimiento (Kiehlhofner, 2004).
Evidencia de esta preocupación es el planteamiento de Weimer (1979, citado en Kielhofner, 2006) "Io nuestro es, y debe ser, el conocimiento básico de la ocupación...Nuestro dominio exclusivo es la ocupación, nosotros debemos refinarla, investigarla y sistematizarla para que se convierta en evidente, definible, defendible y vendible" (p. 65).

El Modelo Ocupación Humana hace énfasis en la ocupación como aspecto central del conocimiento y ejercicio de la Terapia Ocupacional. Surge como alternativa a la teorización y práctica ocupacional de origen biomédico, centrada en procedimientos de rehabilitación de habilidades corporales o mentales. Como refiere KieIhofner (2004), "la ocupación humana es el centro del modelo, buena parte del objetivo de este modelo es proporcionar una explicación de la ocu- 
pación humana según se manifiesta en las vidas individuales" (p. 3).

Este modelo describe y comprende a las personas como seres ocupacionales que se motivan para elegir y realizar ocupaciones significativas, de tal manera que, usando sus capacidades físicas y mentales, las personas se involucran en ocupaciones de manera organizada, en rutinas asociadas a roles ocupacionales, y en un tiempo determinado. El medio ambiente influye en todo este proceso de configuración de la ocupación humana que incluye la integración de la motivación, la organización y la capacidad de realización. Así,

Los tipos de cosas que hacemos, por qué y cómo las hacemos y qué pensamos y sentimos acerca de ellas derivan de las condiciones e influencias de tiempo, espacio, sociedad y cultura que se entrecruzan. La ocupación singularmente humana, que caracteriza a nuestra especie, es una función de estas condiciones e influencias. (Kielhofner, 2004, p.3)

En otras palabras, este modelo presenta la ocupación humana como un proceso integrado. Los componentes de motivación ocupacional, los patrones ocupacionales, las capacidades para las ocupaciones, y el medioambiente interactúan, se influyen mutuamente para producir la adaptación ocupacional. Se da una dinámica de relación y mutua afectación entre los componentes que caracterizan y producen la ocupación humana. "El Modelo Ocupación Humana intenta explicar de qué modo la ocupación es motivada, adopta patrones y es realizada" (Kielhofner, 2004, p. 15).
Sus autores lo proponen como modelo conceptual de la práctica porque plantea una base teórica interdisciplinar amplia e integral desde la Antropología, la Psicología, la Sociología, la Terapia Ocupacional, la Biología, entre otras disciplinas. De este modo, el modelo configura sus elementos y relaciones, a saber: volición, habituación, capacidad de desempeño y medio ambiente. Este modelo ha construido una tecnología para la intervención terapéutica, una serie de recursos para guiar la reflexión y la intervención, en la que se destacan las evaluaciones, los programas y manuales basados en el modelo. En la Figura 2 se presentan los elementos estructurales que caracterizan el modelo.

El modelo presenta un conjunto de conceptos y relaciones a propósito de la ocupación humana. En este se interrelacionan cuatro subsistemas: volición o motivación por la ocupación, habituación o patrón para la ocupación, capacidad o desempeño de la ocupación y medio ambiente. La relación entre estos cuatro subsistemas del ser humano conforma y produce la ocupación humana, que en la dimensión del hacer, se representa en tres niveles crecientes de complejidad: habilidad, desempeño y participación ocupacional. El propósito final de la ocupación humana es la adaptación ocupacional, esta es el producto de la identidad positiva con la ocupación y el logro de la competencia en la realización de la misma.

En el modelo, la motivación por la ocupación o volición es el proceso por el cual las personas se motivan, construyen valores y hacen elecciones 
Figura 2. Proceso de adaptación ocupacional

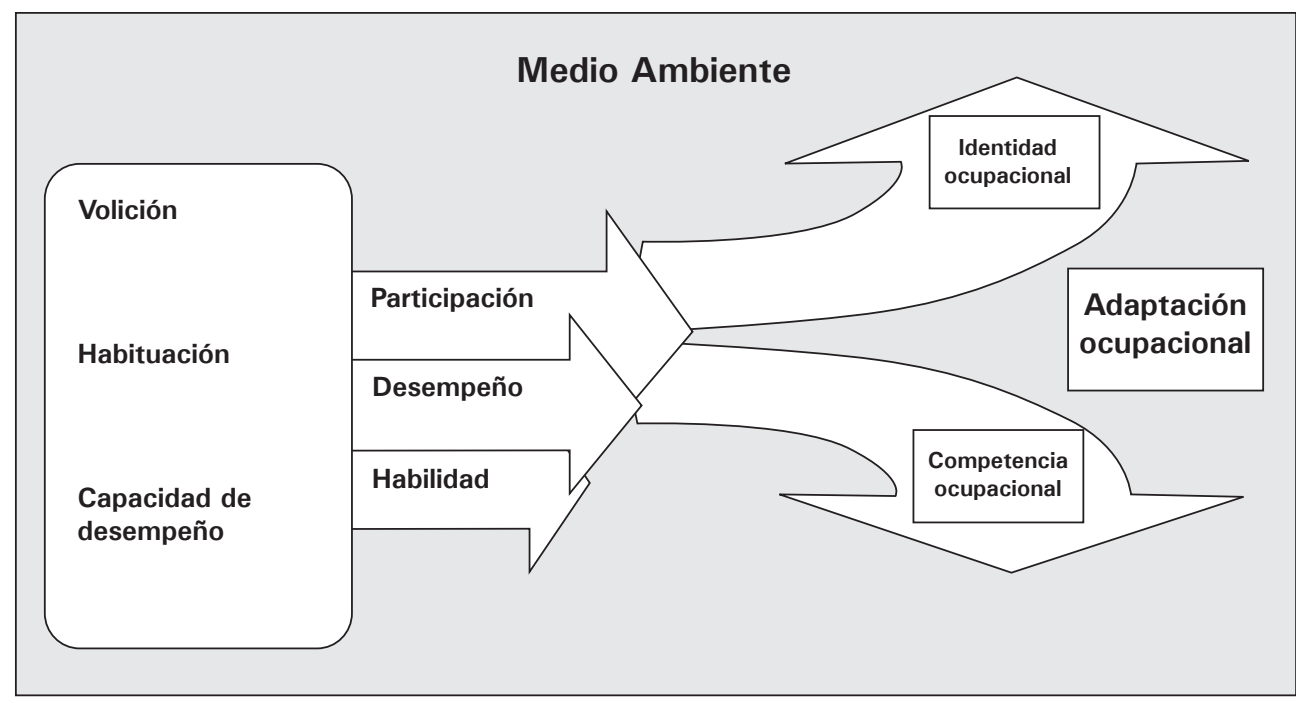

Fuente. Tomado de Kielhofner (2004, p. 139).

ocupacionales. La motivación ocupacional se presenta desde conceptos de la Psicología y la Antropología para comprender las necesidades y las intenciones humanas.

El patrón para la ocupación, también conocido como habituación, es el proceso por medio del cual las ocupaciones se organizan a través de hábitos y rutinas. Para comprender estos elementos se usan conocimientos provenientes de la Terapia Ocupacional, referidos al cuidado de sí mismo, el juego y el trabajo, y de la Sociología de la educación y del trabajo.

El desempeño de la ocupación, o capacidad de desempeño, se refiere a las experiencias y a los recursos físicos y mentales de los sujetos para realizar las ocupaciones. Se comprende desde la fenomenología del cuerpo humano, la unidad cuerpo - mente y el concepto de cuerpo vivido.

El medio ambiente se comprende como el ambiente físico y social en el que se realizan las ocupaciones. Los ambientes pueden ofrecer facilitadores y barreras para el desarrollo de las ocupaciones, a la vez que establecen una serie de demandas para la realización de la ocupación. El medio ambiente se comprende desde conceptos de la Sociología urbana y rural, la Psicología cultural y la Antropología ambiental.

En el Modelo de Ocupación Humana, el hacer ocupacional se compone de tres niveles crecientes de complejidad: el primero es la habilidad, es decir, las diferentes acciones que componen una actividad, por ejemplo, consultar una base de datos para 
la construcción de un proyecto; el segundo es el desempeño, entendido como la realización completa de una actividad, por ejemplo, escribir un proyecto de intervención social para la consecución de recursos; el tercero es la participación, que se refiere a la realización de una ocupación que caracteriza el rol de una persona, por ejemplo, realizar la dirección ejecutiva de una organización no gubernamental.

A medida que las personas adquieren experiencia en la realización de su rol ocupacional en contexto, consiguen la adaptación ocupacional. Esta última se compone de identidad positiva con la ocupación y competencia en la realización de la misma.

En síntesis, el Modelo de Ocupación Humana se construye sobre una base conceptual interdisciplinar para proponer una representación del ser humano, como ser ocupacional. A su vez, construye una tecnología de intervención que permite a los terapeutas ocupacionales describir, comprender y resolver problemas en el hacer de las ocupaciones desde una representación teórica de la ocupación humana.

\section{Discusión}

Recuperando la presentación de los dos modelos de Terapia Ocupacional, tenemos que el Modelo Desempeño Ocupacional Realizante y el Modelo Ocupación Humana son definidos por sus autores como modelos conceptuales. Cada uno de estos propone una estructura conceptual para postular el objeto de estudio de la Terapia Ocupacional, a la vez que presentan una tecnología para la intervención terapéutica (más desarrollada en el Modelo de la Ocupación Humana).

Estos modelos presentan un isomorfismo estructural, es decir, comparten la misma estructura. Son analógicos entre sí porque tal estructura se basa en tres categorías: sujeto, ocupación y medio ambiente, bajo las cuales ubican una serie de conceptos que se relacionan entre sí produciendo, en uno la noción de adaptación ocupacional, y en el otro, la de desempeño ocupacional. Esto evidencia que, los desarrollos específicos de estas dos miradas sobre los componentes internos de cada uno de estos elementos estructurales apunta y aporta énfasis diferentes a la ocupación y al sujeto, como nociones centrales de los correspondientes modelos.

Ahora bien, la noción de sujeto se compone de volición, habituación y capacidad de desempeño en el modelo de Kielhofner (2004), mientras que, de acuerdo con Trujillo (1994), el sujeto está relacionado con la interacción de los aspectos sensoriomotor, cognoscitivo, emocional y social.

Kielhofner conceptualiza al ser humano desde la dimensión de la ocupación; plantea que los seres humanos son seres en tanto se ocupan, ya que la ocupación les da un lugar en la sociedad. Así pues, la ocupación en Kielhofner se asemeja a la razón en Descartes, el trabajo en Marx, o la sexualidad en Freud: un constitutivo fundamental del ser. Para Trujillo, la ocupación constituye un medio para alcanzar como fin la realización humana; al respecto afirma: 
Sin embargo, los marcos de referencia para el ejercicio profesional no incorporan lo que aquí se ha postulado como la meta ulterior de la profesión: de la realización del ser humano a través de la ocupación. Ese es el planteamiento que interesa al Modelo Desempeño Ocupacional Realizante. (Trujillo, 1995, p. 18)

En síntesis, la ocupación en Kielhofner define al sujeto y en Trujillo es un medio que permite la realización del ser humano.

En cuanto a medio ambiente, hay más coincidencia de perspectiva entre los dos autores, en tanto ambos lo conciben como el lugar físico, temporal y social donde ocurre la ocupación, pero también donde es influenciada. Sin embargo, Kielhonfer lo conceptualiza como el proveedor de medios y oportunidades y habla de espacio social y espacio físico. Trujillo lo despliega en términos de lo físico, temporal y sociocultural.

La similitud interna en cuanto a la estructura conformada por un conjunto de conceptos y relaciones que configuran ocupación humana o desempeño ocupacional, identifica estos desarrollos, efectivamente, como modelos dentro del enfoque semántico de las teorías. A continuación se ilustra cómo se cumple esta relación específicamente en el Modelo Desempeño Ocupacional Realizante, entendiendo que lo dicho en este puede aplicarse de modo similar en el Modelo de la Ocupación Humana.

El Modelo de Desempeño Ocupacional Realizante cumple con la característica de ser una idealización, en tanto se presenta como una estructura simbólica formada por los elementos que la componen -procesos humanos, procesos ocupacionales, medio ambiente-, esto sumado a unas relaciones entre estos. El carácter de idealización es el principal requerimiento del enfoque semántico de las teorías para la noción de modelo.

Respecto a lo anterior, cabe agregar que aunque en Terapia Ocupacional se diferencian las nociones de teoría y modelo, en relación con el enfoque semántico, el Modelo Desempeño Ocupacional Realizante cumpliría igualmente la función de teoría, ya que como modelo representa precisamente un aspecto de la realidad constituido por los elementos que relaciona y los conceptos que propone, es decir, actúa como teoría acerca del desempeño ocupacional. Puede afirmarse que se comporta o es asimilable a los modelos representacionales simbólicos de carácter teórico, dentro de la tipología propuesta por la Filosofía de la Ciencia.

De hecho, su construcción tiene como punto de llegada una formulación lingüística en la denominación, definición y enunciación textual de cada uno de los conceptos, se propone como una representación de lo que configura como estructura de dominios y relaciones que interdependen.

Los dos modelos se refieren específicamente a una de las dimensiones y expresiones de la experiencia humana -ocupación y desempeño ocupacional-, y se han configurado a partir de la resolución de hipótesis teóricas acerca de esta experiencia y sus impli- 
caciones en la realización personal de los seres humanos, apoyándose e integrando desarrollos de otras disciplinas.

\section{Conclusiones}

La Terapia Ocupacional concibe los modelos igualmente como representaciones, entidades abstractas, pero no como teorías de principio. Para esta, los modelos son teorías que se ponen en diálogo con la práctica, a propósito de un problema terapéutico, para el diseño de una tecnología que permite darle respuesta, por lo cual se denominan modelos conceptuales de la práctica. Los modelos construyen conocimiento, teoría, aunque no de la misma naturaleza que los modelos teóricos en la ciencia. Modelizar, pues, en ambas miradas, implica conceptualizar, abstraer, resolver proposicionalmente los enunciados de los objetos y las relaciones que se propone representar. Así, en la Filosofía de la Ciencia, por su carácter esencial, los distintos modelos tienen el rasgo implícito de ser conceptuales. $\mathrm{Si}$ en Terapia Ocupacional este rasgo se adjetiviza por su vínculo con la práctica y se hace explícito, obedece a la naturaleza del ejercicio de la profesión que, tal como se ha argumentado, exige no sólo comprensión teórica de los conceptos, sino derivaciones concretas, dispositivos, herramientas para su aplicación en las problemáticas que aborda.

Una característica particular de los modelos analizados es la doble función que cumplen dentro de Terapia Ocupacional, en el sentido de su doble preocupación de modelar, no sólo su objeto de estudio, sino también dispositivos terapéuticos para la intervención profesional. Esto es lo que tratan de resolver, dentro de Terapia Ocupacional, el modelo conceptual de la práctica y el de enfoque teórico, muy similares en su contenido y proyección.

La comprensión de la noción de teoría y de modelo se reconoce como problemática y algo confusa dentro de la literatura de Terapia Ocupacional. Sin embargo, al confrontar los dos modelos analizados con la tipología tomada de la Filosofía de la Ciencia, se pudo establecer que ambos son modelos representacionales simbólicos de carácter teórico. Ambos modelos se definen a partir de una estructura que representa dominios y relaciones sobre su objeto de estudio. Si bien Kielhofner enfatiza como tal la ocupación humana y Trujillo el desempeño ocupacional realizante, ambos construyen su reflexión conceptual considerando las dos nociones.

En consecuencia, el enfoque semántico o modelista en Filosofía de la Ciencia, tomado como referencia para el análisis, resultó particularmente útil para precisar que, más allá de las discusiones internas y los problemas que se atribuyen a la reflexión de Terapia Ocupacional sobre el conocimiento que genera y utiliza, si existe, en los casos presentados, la configuración sólida de los elementos que se requieren cuando se trata de modelar dicho conocimiento.

Esto relativiza las percepciones de imprecisión que casi se han generalizado dentro de la Terapia Ocupacional y llama la atención sobre la potencialidad del tipo de diálogo interdisciplinario propuesto, dados los aportes de 
rigor analítico y categorías de interpretación que, a propósito de lo estudiado, aporta la Filosofía de la Ciencia.

\section{Referencias}

Black, M. (1966). Modelos y arquetipos. Modelos y metáforas. Madrid, España: Técnos.

Giere, R. (1999). Using Models to Represent Reality. En Magnani, L., Nerssesian, N.J. \& Thagard, P. (Ed.), Model -Baset Reasoning in Scientific Discovery (pp. 41-67). New York, EE.UU: Kluwer Academic/Plenum Publisher.

Guerrero, G. (2010). La noción de modelo en el enfoque semántico de las teorías. Praxis filosófica 31, 169-185
Kielhofner, G. (2004). Modelo de la ocupación humana: teoría y aplicación. Buenos Aires, Argentina: Ed. Panamericana.

Kielhofner, G. (2006). Fundamentos conceptuales de la Terapia Ocupacional. Buenos Aires, Argentina: Ed. Panamericana.

Trujillo, A. (1994). Modelo desempeño ocupacional y realización humana: su origen y alcance. Terapia Ocupacional y Universidad (pp.99-112) Bogotá: Universidad Nacional de Colombia.

Trujillo, A. (1995). Desempeño ocupacional realizante: un modelo sobre los propósitos de la Terapia Ocupacional. Ocupación Humana 6 (2), 11-48. 